\title{
A COVERING THEOREM FOR TYPICALLY REAL FUNCTIONS
}

by D. A. BRANNAN and W. E. KIRWAN $\dagger$

(Received 8 June, 1968)

Let $T$ denote the class of functions

$$
f(z)=z+a_{2} z^{2}+\ldots
$$

that are analytic in $U=\{|z|<1\}$, and satisfy the condition

$$
\operatorname{Im} f(z) . \operatorname{Im} z \geqq 0 \quad(z \in U) .
$$

Thus $T$ denotes the class of typically real functions introduced by W. Rogosinski [5].

One of the most striking results in the theory of functions

$$
g(z)=z+b_{2} z^{2}+\ldots
$$

that are analytic and univalent in $U$ is the Koebe-Bieberbach covering theorem which states that $\left\{|w|<\frac{1}{4} \subset g(U)\right.$. In this note we point out that the same result holds for functions in the class $T$, a fact which seems to have been overlooked previously. We also determine the largest subdomain of $U$ in which every $f(z)$ in $T$ is univalent, extending previous results in [1] and [2].

Our basic tool is the following theorem of M. P. Remizova.

THEOREM 1. [3] If $f(z) \in T$ and $z=r e^{i \theta} \in U$, then

$$
|f(z)| \geqq\left\{\begin{array}{lll}
\frac{r}{|1+z|^{2}} & \text { if } & \operatorname{Re}\left(z+\frac{1}{z}\right) \geqq 2, \\
\frac{r}{|1-z|^{2}} & \text { if } & \operatorname{Re}\left(z+\frac{1}{z}\right) \leqq-2, \\
\frac{r\left(1-r^{2}\right)|\sin \theta|}{\left|1-z^{2}\right|^{2}} & \text { if } & \left|\operatorname{Re}\left(z+\frac{1}{z}\right)\right| \leqq 2 .
\end{array}\right\}
$$

We shall also require the following

Lemma. Let $C_{1}$ denote the arc of $|z+i|=\sqrt{2}$ on which $\operatorname{Im} z \geqq 0$. For $z$ on $C_{1}$,

$$
\left|\operatorname{Re}\left(z+\frac{1}{z}\right)\right| \leqq 2 .
$$

Proof. Let $s=\frac{1}{2}(r+1 / r)$, so that $s>1$ for $0<r<1$. If $z \in C_{1}, 2 r \sin \theta=1-r^{2}$, and so $\cos ^{2} \theta=2-s^{2}$. Now $z$ satisfies (2) if and only if $|\cos \theta| \leqq 1 / s$, and this is certainly true for $z$ on $C_{1}$, since $2-s^{2} \leqq s^{-2}$ for all $s \geqq 1$.

$\dagger$ Supported in part by NSF GP 6891. 
TheOREM 2. Let $w=f(z)$ belong to T. Then $\left\{|w|<\frac{1}{4}\right\} \subset f(U)$.

Proof. We assume first that $f(z)$ is continuous in $|z| \leqq 1$. Let $z$ be a point of $C_{1}$ not equal to \pm 1 . By the Lemma and Theorem 1 ,

$$
|f(z)| \geqq \frac{r\left(1-r^{2}\right) \sin \theta}{\left|1-z^{2}\right|^{2}}=\frac{1}{4},
$$

since $2 r \sin \theta=1-r^{2}$ and $\left|1-z^{2}\right|^{2}=1+r^{4}-2 r^{2} \cos 2 \theta=2\left(1-r^{2}\right)^{2}$ for $z=r e^{i \theta}$ on $C_{1}$. On the other hand, it follows from (1) that, for real values of $z$,

$$
|f(z)| \geqq \frac{|z|}{(1+|z|)^{2}} .
$$

Thus, $|f( \pm 1)| \geqq \frac{1}{4}$, and hence $|f(z)| \geqq \frac{1}{4}$ for $z$ on $C_{1}$. If $C_{2}$ denotes the arc of $|z-i|=\sqrt{ } 2$ on which $\operatorname{Im} z \leqq 0$, then $C_{1}$ and $C_{2}$ intersect at 1 and -1 . Since $f(\bar{z})=\overline{f(z)}$ for each $f(z)$ in $T,|f(z)| \geqq \frac{1}{4}$ on $C_{2}$. If we set $C=C_{1} \cup C_{2}$, then we have shown that $|f(z)| \geqq \frac{1}{4}$ on $C$; hence $\left\{|w|<\frac{1}{4} \subset f(U)\right.$ by Rouché's Theorem, since $f(0)=0$.

If $f(z)$ is not continuous in $|z| \leqq 1$, then we apply the above argument to the function

$$
g_{R}(z)=\frac{1}{R} f(R z) \quad(0<R<1) .
$$

Since $\lim _{R \rightarrow 1} g_{R}(z)=f(z)$ for $z$ in $U$, the result follows.

Let $L$ denote the inside of the curve $C$ of the previous theorem; i.e.,

$$
L=\{|z+i|<\sqrt{ } 2\} \cap\{|z-i|<\sqrt{ } 2\} .
$$

In [3], Remizova has shown that the largest subdomain $U^{\prime}$ of $U$ in which every $f(z)$ in $T$ is univalent is contained in $L$. Using a result of L. Cakalov [1], we point out that $U^{\prime}=L$.

THEOREM 3. If $f(z) \in T$, then $f(z)$ is univalent in $L$. Furthermore, if $z_{0} \in U-L$, there is a function in $T$ whose derivative vanishes at $z_{0}$.

Proof. M. S. Robertson [4] has shown that, if $f(z) \in T$, then

$$
f(z)=\int_{-1}^{1} \frac{z}{1-2 t z+z^{2}} d \alpha(t)=\frac{1}{2} \int_{-1}^{1} \frac{d \alpha(t)}{w-t},
$$

where $\alpha(t)$ is increasing on $[-1,1], \alpha(1)-\alpha(-1)=1$, and $w=\frac{1}{2}(z+1 / z)$. In [1], Čakalov proved that $\int_{-1}^{1} \frac{d \alpha(t)}{w-t}$ is univalent in $\{|w|>1\}$. The curve $|w|=1$ is the image of $C$ under the transformation $w=\frac{1}{2}(z+1 / z)$, and, consequently, $f(z)$ is univalent in $L$.

Finally, for any $\lambda(0<\lambda<1)$, the function defined by

$$
f_{\lambda}(z)=\frac{\lambda z}{(1-z)^{2}}+\frac{(1-\lambda) z}{(1+z)^{2}}
$$


is in $T$, since $T$ is a convex set containing $z /(1 \pm z)^{2}$. Now $f_{\lambda}^{\prime}(z)=0$ when

$$
\frac{1+z}{1-z}=\left(-\frac{1-\lambda}{\lambda}\right)^{1 / 4},
$$

and as $z$ varies round $C,\{(1+z) /(1-z)\}^{4}$ attains all real negative values; hence, if $z_{0}$ is a point of $U$ which lies on $C, \lambda$ can be chosen so that $f_{\lambda}^{\prime}\left(z_{0}\right)=0$. If $z_{0}$ is any point of $U-L$, then, by considering the function $R^{-1} f_{\lambda}(R z)(0<R<1)$, we obtain a function in $T$ whose derivative vanishes at $z_{0}$.

Note. In [2] it was shown that any function in $T$ maps $\{|z|<\sqrt{ }(2)-1\} \subset L$ univalently onto a domain starlike with respect to $w=0$.

\section{REFERENCES}

1. L. Čakalov, Maximal domains of univalency of some classes of analytic functions, Ukr. Math. J. 11, no. 4 (1959), 408-412 (Russian).

2. W. E. Kirwan, Extremal problems for the typically real functions, Amer. J. Math. 88 (1966), 942-954.

3. M. P. Remizova, Extremal problems in the class of typically-real functions, Izv. Vyss. Uc. Zaved. Mat. (1963), no. 1 (32), 135-144.

4. M. S. Robertson, On the coefficients of typically real functions, Bull. Amer. Math. Soc. 41 (1936), 565-572.

5. W. W. Rogosinski, Über positive harmonische Entwicklungen und typische-reelle Potenzreihen, Math. Z. 35 (1932), 93-121.

UNIVERSITY OF MARYLAND

College Park, MD., U.S.A. 